\title{
Early Detection of Exacerbation of the Severe Acute Respiratory Syndrome Coronavirus 2 Infection using Fitbit (DEXTERITY Pilot Study)
}

\section{Kan Yamagami}

Department of Cardiovascular Medicine, Kanazawa University Graduate School of Medical Sciences, Kanazawa, Japan https://orcid.org/0000-0002-4794-0679

Akihiro Nomura ( $\nabla$ anomura@med.kanazawa-u.ac.jp )

Department of Cardiovascular Medicine, Kanazawa University Graduate School of Medical Sciences, Kanazawa, Japan https://orcid.org/0000-0001-6647-8240

\section{Mitsuhiro Kometani}

Department of Health Promotion and Medicine of the Future, Kanazawa University Graduate School of Medical Sciences, Kanazawa, Japan

\section{Masaya Shimojima}

Department of Cardiovascular Medicine, Kanazawa University Graduate School of Medical Sciences, Kanazawa, Japan

\section{Kenji Sakata}

Department of Cardiovascular Medicine, Kanazawa University Graduate School of Medical Sciences, Kanazawa, Japan

\section{Soichiro Usui}

Department of Cardiovascular Medicine, Kanazawa University Graduate School of Medical Sciences, Kanazawa, Japan

\section{Kenji Furukawa}

Health Care Center, Japan Advanced Institute of Science and Technology, Ishikawa, Japan

\section{Masayuki Takamura}

Department of Cardiovascular Medicine, Kanazawa University Graduate School of Medical Sciences, Kanazawa, Japan

\section{Masaki Okajima}

Intensive Care Unit, Kanazawa University Hospital, Kanazawa, Japan

\section{Kazuyoshi Watanabe}

JCHO Kanazawa Hospital, Kanazawa, Japan

\section{Takashi Yoneda}

Department of Health Promotion and Medicine of the Future, Kanazawa University Graduate School of Medical Sciences, Kanazawa, Japan 


\section{Research Article}

Keywords: COVID-19,Fitbit, smartwatch, silent hypoxia

Posted Date: February 2nd, 2021

DOl: https://doi.org/10.21203/rs.3.rs-156774/v1

License: (c) (i) This work is licensed under a Creative Commons Attribution 4.0 International License. Read Full License

Version of Record: A version of this preprint was published at European Heart Journal on October 1st, 2021. See the published version at https://doi.org/10.1093/eurheartj/ehab724.3089. 


\section{Early Detection of Exacerbation of the Severe Acute Respiratory Syndrome Coronavirus 2 Infection using Fitbit (DEXTERITY Pilot}

\section{Study)}

Kan Yamagami ${ }^{1,2 \#}$ Akihiro Nomura ${ }^{1,3,4 * \#}$, Mitsuhiro Kometani ${ }^{5}$, Masaya Shimojima ${ }^{1}$, Kenji Sakata ${ }^{1}$, Soichiro Usui ${ }^{1}$, Kenji Furukawa ${ }^{6}$, Masayuki Takamura $^{1}$, Masaki Okajima ${ }^{7}$, Kazuyoshi Watanabe², Takashi Yoneda ${ }^{5}$

\#: These authors contributed equally

\section{Affiliations:}

1. Department of Cardiovascular Medicine, Kanazawa University Graduate School of Medical Sciences, Kanazawa, Japan

2. JCHO Kanazawa Hospital, Kanazawa, Japan

3. Innovative Clinical Research Center, Kanazawa University, Kanazawa, Japan

4. CureApp Institute, Karuizawa, Japan

5. Department of Health Promotion and Medicine of the Future, Kanazawa University Graduate School of Medical Sciences, Kanazawa, Japan

6. Health Care Center, Japan Advanced Institute of Science and Technology, Ishikawa, Japan

7. Intensive Care Unit, Kanazawa University Hospital, Kanazawa, Japan 
*Corresponding author:

Akihiro Nomura, MD, PhD

Associate Professor

Innovative Clinical Research Center

Department of Cardiovascular Medicine

Kanazawa University Graduate School of Medical Sciences

13-1 Takaramachi Kanazawa, Ishikawa, Japan

E-mail: anomura@med.kanazawa-u.ac.jp 


\begin{abstract}
Some patients with coronavirus disease 2019 (COVID-19) experienced sudden death because of sudden symptom deterioration. Thus, an alarm system that could detect early signs of COVID-19 exacerbation beforehand, to prevent serious illness or death of patients while receiving outpatient treatment at home or in hotels is necessary. Here, we tested whether estimated oxygen variations (EOV), a relative physiological scale that represents users' blood oxygen saturation level during sleep measured by Fitbit, predicted COVID-19 symptom exacerbation. Study period was from August to November 2020. We enrolled 23 COVID-19 patients diagnosed by SARS-CoV-2 polymerase chain reaction-positive (mean age \pm standard deviation, $50.9 \pm 20$ years; $70 \%$ female), let each patient wore the Fitbit for 30 days; COVID19 symptoms were exacerbated in $6(26 \%)$. High EOV signal (a patient's oxygen level exhibits significant dip and recovery within the index period) had $80 \%$ sensitivity before symptom exacerbations, whereas resting heart rate signal only had $50 \%$ sensitivity. Coincidental obstructive sleep apnea syndrome confirmed by polysomnography was detected in a patient by consistently high EOV signals. This pilot study successfully detected early COVID-19 symptoms exacerbation by measuring EOV and may help to identify early signs of COVID-19 exacerbation.
\end{abstract}




\section{Introduction}

The coronavirus disease 2019 (COVID-19) pandemic caused by severe acute respiratory syndrome coronavirus 2 (SARS-CoV-2) has resulted in over 80 million cases and 1.8 million deaths worldwide as of early January $2021 .^{1}$ The virus, with a long latency of 2-14 days from initial infection to the onset of symptoms, can be most transmitted just before the symptoms appear. ${ }^{2}$ Approximately half of infected patients are asymptomatic, but can spread the infection to others. Therefore, being asymptomatic might be one of the strong factors for the global explosion of the infection. ${ }^{3}$

A feature of COVID-19 includes that a certain number of patients experience a rapid deterioration after 1 week of initial symptom onset - requiring oxygen or care in the intensive care unit (ventilators and extracorporeal membrane oxygenation [ECMO]) for severe pneumonia and acute respiratory distress syndrome-like symptoms. ${ }^{4}$ According to a New York City report, the mortality rate of COVID-19 for patients on ventilators is over $75 \% .^{5}$ The benefit of antiviral medication such as remdesivir or dexamethasone might be the most apparent when it is used before symptom exacerbation. ${ }^{6,7}$ Thus, it is important to predict COVID-19 symptom exacerbation at an early stage before the experience of increasing severity in patients. In Japan, an increasing number of COVID-19 patients with mild symptoms are managed in their homes or hotels to make effective use of medical resources. ${ }^{8}$ However, some tragic news revealed that symptoms in some COVID-19 patients deteriorated and they died before reaching a hospital. ${ }^{9,10}$ Therefore, it is necessary to construct an alarm system to detect signs of severity beforehand, to prevent patients from serious illness or death while waiting at home or in the hotels.

To evaluate the severity of pulmonary diseases, blood oxygen saturation $\left(\mathrm{SpO}_{2}\right)$ levels measured by pulse oximetry, usually provides important information. ${ }^{11}$ In fact, patients with 
COVID-19 could experience a unique "silent hypoxia" at an early stage of the infection when they are apparently asymptomatic, but with a rather low $\mathrm{SpO}_{2}$ levels. ${ }^{12}$ Since a low $\mathrm{SpO}_{2}$ normally indicates a severe pulmonary involvement of the disease, monitoring of $\mathrm{SpO}_{2}$ could provide potential biometric data to predict impending disease deterioration. ${ }^{13}$ Indeed, low $\mathrm{SpO}_{2}$ level could predict future disease exacerbation in patients with chronic obstructive pulmonary disease. ${ }^{11}$ However, except in special situations in an intensive care unit, continuous $\mathrm{SpO}_{2}$ monitoring in daily life is unlikely because the measuring equipment normally needs to be clipped to one's finger for every measurement.

Recently, a high-performance wearable device such as Apple Watch or Fitbit has become commercially available, that monitors several data including steps, resting heart rates (RHRs), physical activity, sleep quality, and even estimated oxygen variations (EOV; a relative physiological scale that reflects users $\mathrm{SpO}_{2}$ levels during sleep). For example, one study used biometric data obtained from a Fitbit, a smartwatch device worn on one's wrist, and showed that increased RHR and decreased sleep duration were associated with flu-like symptoms. ${ }^{14}$ With COVID-19, some studies demonstrated that changes in certain biometric data including RHRs, sleep durations, or respiratory rates, from the baseline, might predict the occurrence of COVID-19 symptoms before its onset, for those who use wearable devices on a daily basis. ${ }^{15-17}$ However, it is still unclear whether the detection of blood oxygen variations might be one of the key components for predicting COVID-19 severity by wearable devices, or whether wearable devices can detect the signs of exacerbation of COVID-19 symptom in confirmed COVID-19 patients.

Here, we conducted the "DEXTERITY" (Early Detection of Exacerbation of the Severe Acute Respiratory Syndrome Coronavirus 2 Infection using Fitbit) pilot study leveraging a 
wearable device, to obtain biometric data, especially EOV and RHRs, in patients diagnosed with COVID-19, to predict symptom exacerbation.

\section{Results}

Study participants and baseline characteristics

We prospectively recruited 28 SARS-CoV-2 polymerase chain reaction ((PCR)-positive patients in this pilot study. Of those, four patients did not link to the Fitbit account, and one patient had no personal email address. Therefore, 23 patients were followed-up for 30 days and included in further analyses (Figure 1).

Figure 1. Study flowchart.

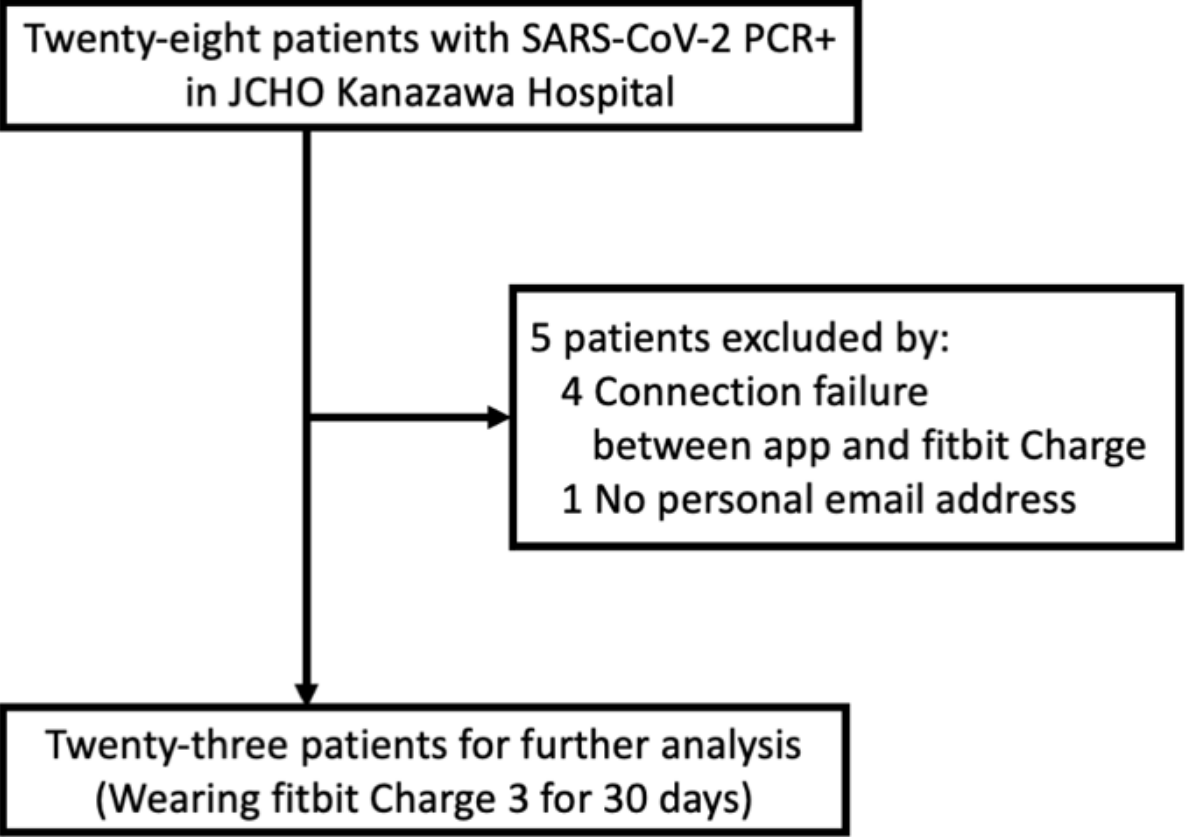


Table 1 shows the baseline characteristics of the patients. A total of 23 patients were included (mean age \pm standard deviation, $50.9 \pm 20$ years; 70\% female). Four patients (17\%) had medical history of malignancy, but none with cardio or cerebrovascular diseases. Symptoms at admission was dyspnea in 2 patients (9\%), fever in 12 patients (52\%), dysgeusia in 3 patients (13\%), dysosmia in 1 patient (4\%), sore throat in 1 patient (4\%), and no symptoms in 4 patients (17\%). Median interval from initial COVID-19 symptoms to wearing of Fitbit was 5 days (range, 1 to 9 days), and median days of wearing Fitbit were 19 (interquartile range [IQR], 15.5 to 28$)$ days. 
Table 1. Baseline characteristics

Total $\mathrm{N}=23$

\begin{tabular}{lr}
\hline Age (years), mean \pm SD & $50.9 \pm 20$ \\
Female, $\mathrm{n}(\%)$ & $16(70)$ \\
Body weight $(\mathrm{kg})$, mean $\pm \mathrm{SD}$ & $58.7 \pm 16$ \\
BMl $\left(\mathrm{kg} / \mathrm{m}^{2}\right)$, mean $\pm \mathrm{SD}$ & $22.8 \pm 4.7$
\end{tabular}

Comorbidities

Hypertension, n (\%) $\quad 5(22)$

Diabetes mellitus, n (\%) $\quad 2$ (9)

Dyslipidemia, n (\%) $\quad 5(22)$

Medical history, $\mathrm{n}$ (\%)

Malignancy $4(17)$

Cardio- or cerebrovascular diseases $\quad 0(0)$

Smoking status, n (\%)

Never $12(52)$

Former 9 (39)

Current 2 (9)

Symptoms at admission, $\mathrm{n}(\%)$

Fever $12(52)$

Dysgeusia $3(13)$

Dyspnea 2 (9)

Dysosmia 1 (4)

Sore throat 1 (4) 
SD, standard deviation 


\section{Estimated oxygen variation and outcomes}

Figure 2 demonstrates the summary of observed high EOV signals among the 23 patients. We observed 48 high EOV signals (73 single-day high signals) during the study. We found a median high EOV signal number per patient of 1 (IQR, 1 to 3). The median percentage of EOV per day was $16 \%$ (IQR, 11\% to 19\%). Of the 23 patients, we excluded one patient (ID-023) from further analyses because of obstructive sleep apnea syndrome (OSAS) detected at polysomnography (PSG) (details are described in Representative case 3).

The primary outcomes (symptom exacerbation events) occurred seven times in six patients during the study period. The primary outcomes included the use of high flow nasal cannula (HFNC) ( $n=2)$, exacerbation of cough ( $n=2 ; 1$ patient was re-admitted to the hospital), exacerbation of dysosmia $(n=1)$, and experience of fever and general malaise $(n=1)$. In patients with EOV data, we successfully observed high EOV signals within 2 days of the symptom exacerbation events in 4 of 5 cases (sensitivity, 80\%; specificity 90\%). Although negative prediction value (NPV) was 99.7\%, positive prediction value (PPV) was just 9.3\%. The reference sensitivity, specificity, PPV, and NPV of high RHR signals for detecting these events were $50 \%$, $88 \%, 6.7 \%$, and $99.1 \%$ respectively, all of which were lower than those of high EOV signals. All clinical course of the COVID-19 patients were shown in Supplementary Figure 1.

Figure 2. Summary of high EOV signals and events among SARS-CoV-2 PCR-positive patients The vertical columns represent the assigned identity numbers (ID) of each patient, and the horizontal axis represent the number of days after wearing Fitbit. Blue squares represent a single day high EOV signal. Gray columns denote a day of no EOV data. Red columns indicate the days that the primary outcomes (symptom exacerbation events) occurred. 


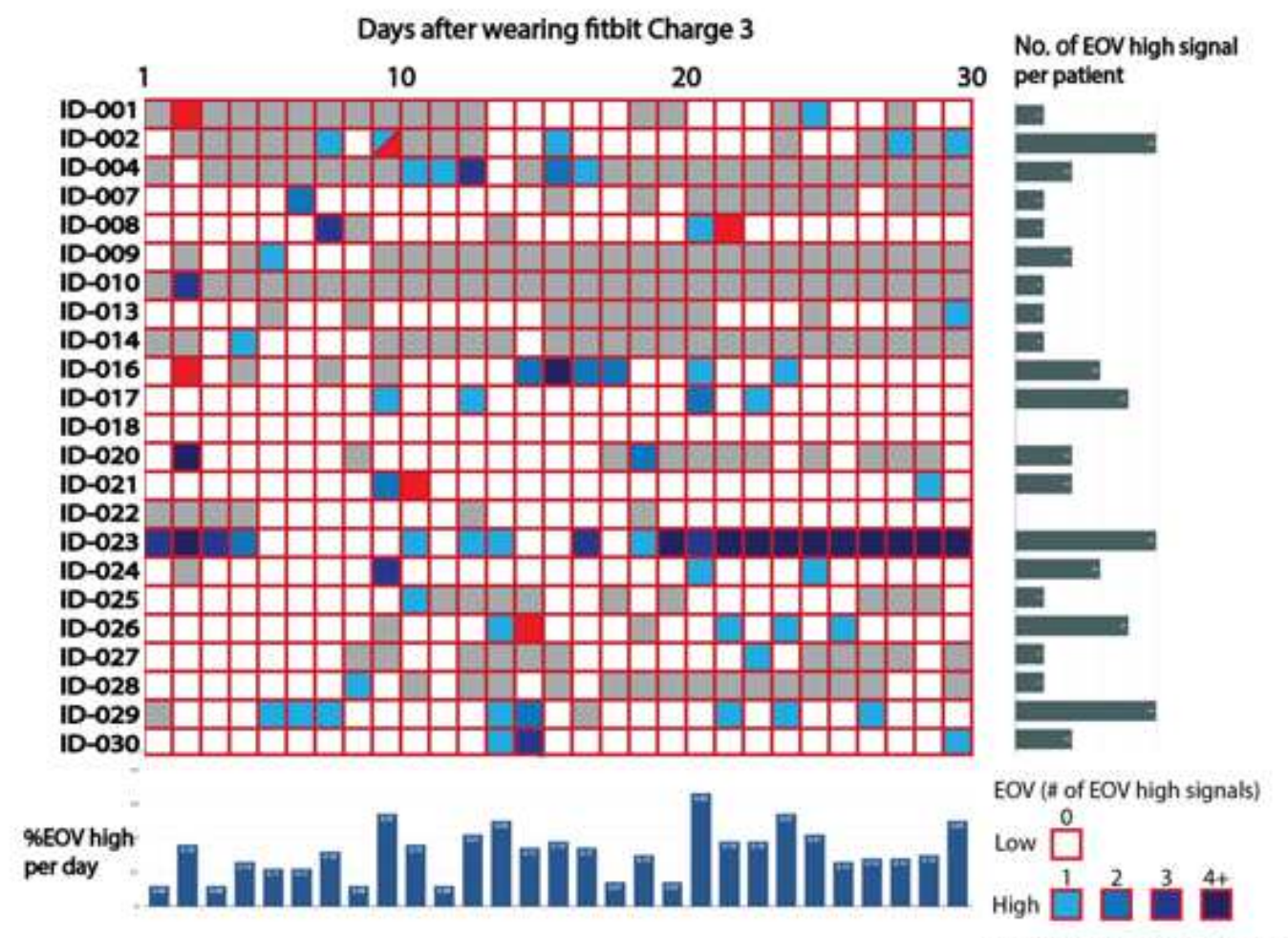

Onset of symptom exacerbation

Next, we reported representative cases in whom we could successfully observe (ID-008) or not observe (ID-016) high EOV signals just before the exacerbation of COVID-19 symptoms (Figure 3). Also, we presented a COVID-19 patient (ID-023) in whom we unintentionally detected a remarkable case of OSAS by showing extreme and consistently high EOV signals during the study period.

Figure 3. The clinical course of COVID-19 in ID-008 (a), ID-016 (a), and ID-023 (C).

The vertical columns represent EOV, RHR (compared with minimum RHR), body temperature, and cough or fatigue severity levels. The horizontal axis represents the number of days after 
positive SARS-CoV-2 PCR test (ID-008) or the occurrence of COVID-19-related symptoms (ID-

016 and ID-023).

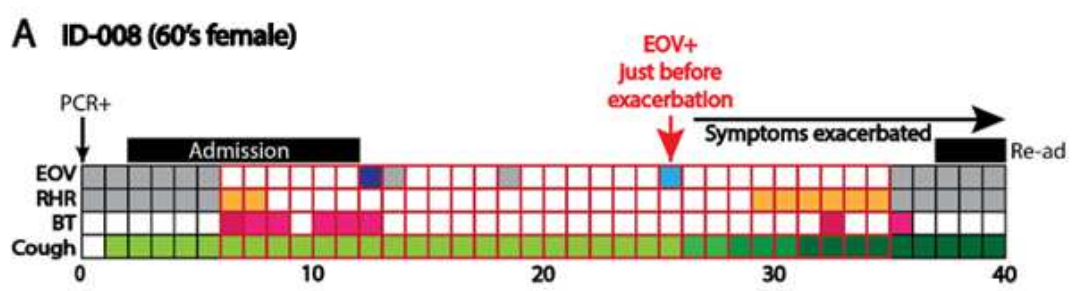

\section{B ID-016 (70'smale)}

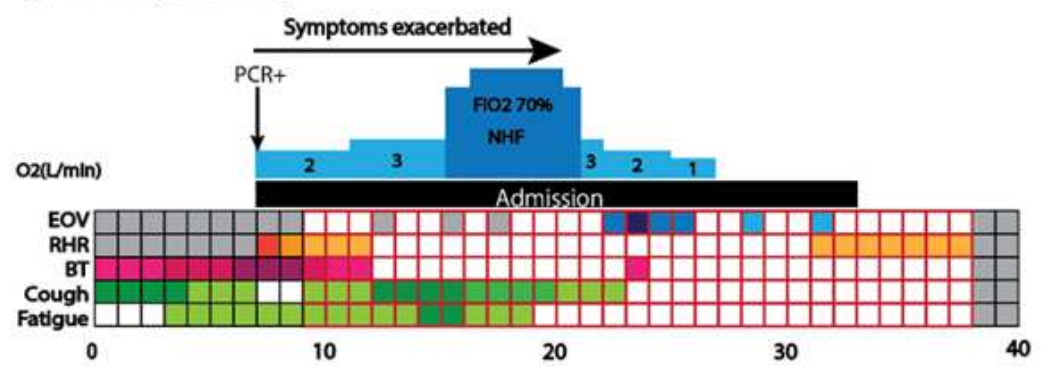

\section{ID-023 (50's male)}

Fitbit equipped $\square$

EOV (\# of EOV high signals)

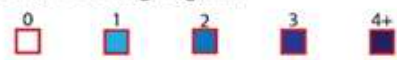

RHR (Compared with minimum RHR)

$0-5 \quad+6-10 \quad+11-15+16-20>+21$ BT (Body temperature) ( $\left.{ }^{\circ} \mathrm{C}\right)$

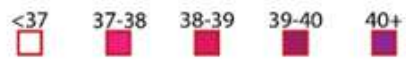

Cough / Fatigue

None Mild Moderate Severe Very severe

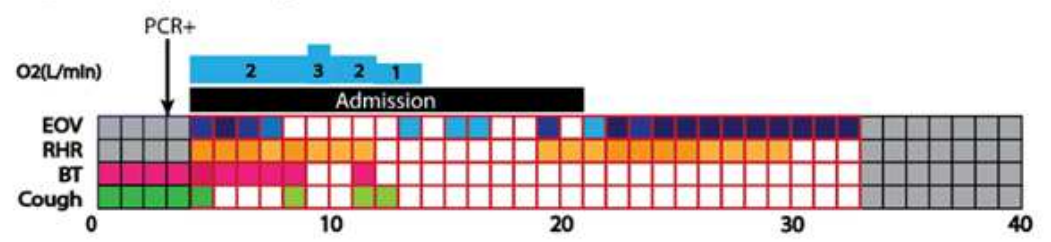

Days after the onset of Inittal COVID-19 symptom or SARS-CoV-2 PCR+ 
Representative case 1 (successfully detected): ID-008

A 60's woman with a history of gastric cancer was referred to our hospital due to positive SARS-CoV-2 PCR test result (Figure 3a). She was asymptomatic at the time of diagnosis by PCR. However, she started coughing just before the hospital admission, and her $\mathrm{SpO}_{2}$ was $98 \%$ at room air. Although she experienced moderate fever (up to $38.8^{\circ} \mathrm{C}$ ) with computed tomography (CT)-confirmed pneumonia for a week, she became afebrile and asymptomatic except for a slight cough on the day of discharge at day 12 (d12). High EOV signal was once detected just after the discharge but no symptom exacerbation occurred after the signal. However, another high EOV signal occurred on d25. Since her cough was suddenly exacerbated following fever after the signal, she visited a hospital on d30. Her chest CT exhibited COVID-19-like infiltration and interstitial shadow, and she was readmitted to the hospital and diagnosed with COVID-19-induced pneumonia recurrence. In this case, high EOV signal at d25 was successfully observed just before the symptom exacerbation from $\mathrm{d} 26$.

Representative case 2 (undetected): ID-016

We here present an undetectable case of EOV for COVID-19 symptom exacerbation before symptom onset. A 70's man with a history of hypertension was referred to our hospital due to positive SARS-CoV-2 PCR test (Figure $3 \mathbf{b}$ ). At admission, his body temperature was $39.6^{\circ} \mathrm{C}$, $\mathrm{SpO}_{2}$ of $98 \%$ (via $\mathrm{O}_{2}$ nasal canula at a rate of $2 \mathrm{~L} / \mathrm{min}$ ), and his chest $\mathrm{CT}$ showed no shadow compatible with COVID-19. However, his $\mathrm{SpO}_{2}$ gradually worsened and he had to use HFNC to maintain oxygenation on $\mathrm{d} 9$ (maximum fraction of inspired oxygen $\left.\left[\mathrm{FiO}_{2}\right], 70 \%\right)$. Chest $\mathrm{CT}$ on d8 revealed ground glass opacity (GGO) compatible with COVID-19. During this exacerbation, no high EOV signal was observed. However, just after HFNC discontinuation on d13, high EOV signals were observed daily from $\mathrm{d} 14$ to $\mathrm{d} 17$, but no exacerbation of symptoms was found 
afterwards. Chest CT on d15 showed remarkable improvement of GGO. He discontinued oxygenation on d21 and was discharged from the hospital on d28. After discharge, high EOV signals were detected twice, but again, no exacerbations of any symptoms were found after these signals.

Representative case 3 (obstructive sleep apnea syndrome): ID-023

We present a COVID-19 patient in whom OSAS was coincidentally detected by consistently high EOV signals during the study period. A 50's man with a history of hypertension was referred to our hospital due to positive SARS-CoV-2 PCR test (Figure 3c). He experienced mild fever (up to $37.8^{\circ} \mathrm{C}$ ) and moderate cough ( 4 days before admission). At admission, his body temperature was $38.4^{\circ} \mathrm{C}, \mathrm{SpO}_{2}$ of $96 \%\left(\mathrm{O}_{2}\right.$ nasal canula, $\left.2 \mathrm{~L} / \mathrm{min}\right)$, and his chest $\mathrm{CT}$ showed shadows compatible with COVID-19. Just after admission, high EOV signals were observed from $\mathrm{d} 1$ to $\mathrm{d} 4$, but no exacerbation of symptoms was found after these signals. His body temperature and cough improved gradually after admission, and he became afebrile and asymptomatic after $\mathrm{d} 9$. His $\mathrm{SpO}_{2}$ also improved gradually, and he could discontinue oxygenation on d11. High EOV signals were observed on $\mathrm{d} 10, \mathrm{~d} 12-13$, and $\mathrm{d} 16$, but no symptom exacerbation occurred after these signals. He was discharged from the hospital on d17 and had been afebrile and asymptomatic thereafter. However, a long-lasting high EOV signal was observed from d18 to d29 without any symptoms. At this time, we assumed that he might be having sleep apnea syndrome (SAS). He underwent polysomnography by a portable polysomnogram monitor (SAS-2200, NIHON KOHDEN, TOKYO), and his Oxygen Desaturation Index 3\% (3\%ODI) was 33.6/h; indicating severe OSAS. In this case, high EOV signal was observed not by the exacerbation of COVID-19 symptoms but by the existing OSAS. 


\section{Discussion}

This is the first prospective pilot study to assess whether EOV, a relative physiological scale that indicates continuous $\mathrm{SpO}_{2}$ variations during sleep by a Fitbit wearable device, could predict early exacerbation signs of SARS-CoV-2 infection before its onset. We demonstrated that the high EOV signals observed just before the symptom exacerbations in four out of five cases (80\%) was higher than that in RHR signals. In addition, we detected a severe case of OSAS by the intermittently high EOV signals obtained from the Fitbit device.

This study provided several findings. First, the high EOV signal by Fitbit demonstrated a favorable sensitivity (80\%) and high NPV (99.7\%) (both higher than those of RHR signals) for COVID-19 symptom exacerbations before their onset. High sensitivity and NPV of the device and signal used in this study are of particular importance for screening/early diagnosis of COVID-19 exacerbation that could accurately identify all possible cases that could warrant closer inpatient or outpatient monitoring. In some patients with COVID-19, silent hypoxia has been reported, with remarkably low $\mathrm{SpO}_{2}$ levels, while having minimal typical symptoms such as fever, cough, or fatigue. ${ }^{18}$ Following the experience of silent hypoxia, the patients had apparent symptoms. ${ }^{19,20}$ The mechanism of silent hypoxia involves a combination of many factors including the response of the respiratory centers and effect of comorbidity (e.g. diabetes mellitus) and older age on breathing control. ${ }^{12}$ Additionally, the idiosyncratic action of coronavirus on receptors involved in chemosensitivity to oxygen has been implicated. ${ }^{12}$ Actually, angiotensin-converting enzyme 2, the cell receptor of SARS-CoV-2, is expressed in the carotid body, the site in which the chemoreceptors sense oxygen. ${ }^{21}$ The development of a thrombi within the pulmonary vasculature may also be related to silent hypoxia. ${ }^{20}$ In this study, since silent hypoxia-like sharp $\mathrm{SpO}_{2}$ depletion without apparent symptoms occurred in some patients, the EOV could successfully predict the exacerbation of COVID-19 symptoms. 
Second, although high EOV signals showed high sensitivity and NPV for detecting COVID-19 symptom exacerbation, PPV of high EOV signals was just 9.3\%. High EOV signals may be invoked not only by $\mathrm{SpO}_{2}$ exacerbation but also by other situations including alcohol intake, emotional stress events, or medications, as the heart rates increase in such situations. ${ }^{15}$ From recent studies predicting onset of COVID-19, RHR- and sleep duration-derived indices tended to be falsely positive after an experience of COVID-19. ${ }^{15,17}$ The same situation might also have occurred for EOV, and we still need to take multiple factors to account that could interfere with the high EOV signal in predicting COVID-19 symptom exacerbation.

Third, we detected a case of severe OSAS in a patient by chance, from the unusual consistently high EOV signals. To our knowledge, this is the first study to report a clinical case of extremely high EOV signals obtained by the Fitbit, to detect SAS. Obstructive sleep apnea is a common disorder that causes the patients to temporarily stop or decrease their breathing repeatedly during sleep. ${ }^{22}$ It is caused by a dynamic upper airway collapse, and demonstrate low $\mathrm{SpO}_{2}$ during the night. ${ }^{23}$ We consider that these $\mathrm{SpO}_{2}$ depletion events were detected by Fitbit as high EOV signals. Now, Fitbit Inc. is applying to the United States Food and Drug Administration to include this EOV function in a medical device to diagnose SAS. Once approved, Fitbit may soon become an innovative device to diagnose SAS.

This study has several limitations. First, Fitbit, its functions, and analysis algorithms including the EOV was not yet approved as a medical device as at the time of this study. Second, we could not acquire sufficient baseline data for each patient regarding RHR, EOV, and other biometric data because the study period when the patients wore Fitbit was only 30 days. Baseline biometric data of each physiological metric are very important to distinguish abnormal signals from normal variations at the individual level. However, COVID-19 patients in this study wore the Fitbit for only 30 days, that is not normally sufficient to obtain baseline 
data from each patient. In this study, we set the minimum value of each biometric factor during the study as the baseline value, but this could have affected the evaluation of positive signal for each metric. Third, we could not assess sleep duration as one of the biometric markers for the exacerbation detection. We tried to investigate the associations of each sleep duration per day with the exacerbation of COVID-19 symptoms, but had to abandon it because of the lack of baseline data and significant variations between in-hospital and after-discharge periods. Fourth, elderly patients who could not use a smartphone daily did not participate in this study, although they were more likely to have exacerbated SARS-CoV-2 infection symptoms.

In conclusion, we demonstrate that EOV from the Fitbit wearable device could detect $80 \%$ of exacerbations of the early signs of SARS-CoV-2 infection before their onset. Additionally, we coincidentally detected OSAS by obtaining consistently high EOV signals. In future, we hope to integrate EOV and other physiological metrics such as RHR, respiratory rates, or sleep data to improve the prediction accuracy of COVID-19 symptom exacerbations in advance. 


\section{Methods}

\section{Participants}

We prospectively recruited 28 SARS-CoV-2 PCR-positive COVID-19 patients for this study, according to the study protocol approved by the Kanazawa University and the Japan Health Care Organization (JCHO) Kanazawa Hospital Institutional Review Boards. This study was conducted from August to November 2020 in JCHO Kanazawa Hospital, Kanazawa, Japan. We performed the study in compliance with the Ethical Guidelines for Medical and Health Research Involving Human Subjects, the Declaration of Helsinki, and other guidelines in Japan. We registered this study with the University Medical Information Network Clinical Trial Registry (UMIN000041421).

We included patients who were diagnosed with COVID-19 and had a positive SARSCoV-2 PCR test result within 1 week before enrollment in this study. We excluded patients who met the following criteria: 1) unable to wear and use the wearable device; 2) unable to connect the wearable device with the smartphone app; 3) unable to download or use the smartphone app; and 4) unable to provide informed consent because of severe COVID-19 symptoms. We obtained an electronic informed consent (eConsent) via mobile platforms using the Research Electronic Data Capture (REDCap) system, from all the study participants.

\section{Wearable device and data extraction}

We provided a Fitbit Charge 3 to each study participant. They wore the wearable device for 30 days to detect COVID-19 symptom exacerbation that normally occurs at 7 to 14 days after the onset of the initial symptom. ${ }^{4}$ The Fitbit Charge 3 was connected to each patient's smartphone via the Fitbit app, and their biometric data including RHR and sleep were extracted through the fitabase, a web-based Fitbit-derived data extraction system for a clinical 
study.

Patients were asked to complete electronic questionnaires in the REDCap system including their baseline characteristics (age, sex, height, weight, BMI, smoking status [current, former, or never], presence of hypertension, diabetes mellitus or dyslipidemia, any other medical history, and any medication use) at the time of enrollment. Additionally, we obtained information regarding COVID-19-related symptoms from the patients during hospitalization and after discharge, during the 30-day study period. The COVID-19-related symptoms included fever, cough, fatigue, difficulty in breathing, nausea, diarrhea, dysosmia, or dysgeusia. We also checked weekly, if they had relief or worsening (exacerbation) of these symptoms, readmission to the hospital, oxygenation, use of mechanical ventilation, use of ECMO, or all cause death, via telephone by the study investigators.

\section{Estimated oxygen variation}

In addition to the biometric data obtained from the Fitbit Charge 3, we directly collected daily EOV graphs by taking screenshots of each patient's app. EOV is internally calculated using a certain algorithm that estimates the variation in the reflected rate from the reflected optical signals, every minute. If a patient's oxygen level is stable, the variation is low or close to zero. However, if a patient's oxygen level exhibits significant dip and recovery within the index period, the variation shows a high signal. Variations are shown above the threshold in Figure 4. We defined high EOV (single day) signal as when the EOV graph exceeded the threshold line by one or more times, during sleep. Since we considered that a symptom deterioration signal could last for several days, if a high single-day EOV signal continues for 2 or more consecutive days, we regarded the signal as a high EOV signal to calculate the sensitivity, specificity, PPV, and NPV. 
Figure 4. Representative graphs of low (negative) and high (positive) EOV signals

\section{Low \\ EOV signals}

\section{High \\ EOV signals (+)}

No. of high signals
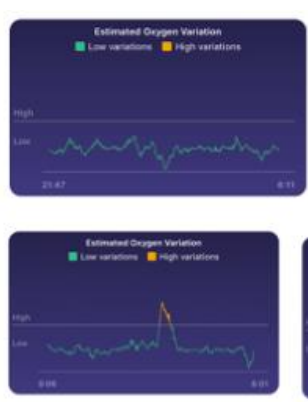

$1+$

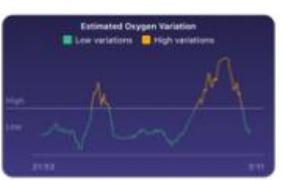

2+

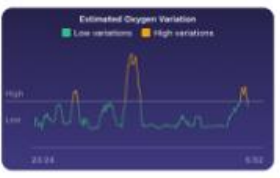

3+

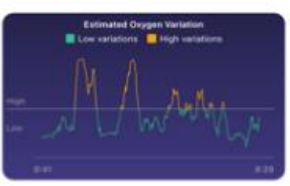

4 or more

\section{Outcomes}

The primary outcome was the composite of all cause death, use of ECMO, use of mechanical ventilator, oxygenation, and the exacerbation of COVID-19 symptoms, irrespective of readmission. We also assessed each outcome individually as secondary outcomes. We made phone call to each patient every week, and asked whether their symptoms were stable or changed. If the patient's status of COVID-19 symptoms changed, we ask the patient when it occurred, and two study investigators judged if the change was deemed to the COVID-19 symptom exacerbation or not.

\section{Statistical analysis}

The baseline profile is shown as the mean \pm standard deviation, or median with quantiles (for continuous variables), or proportions (for categorical variables). We overlayed and compared the onset of the outcomes and the days of detecting high EOV signals. We defined a success of predicting an outcome by EOV as the presence of high EOV signal(s) within 2 days of the onset of the outcome. We also defined high RHR signals as daily RHR exceeding 5 beats/day compared with the minimum RHR of each patient during the study period. We calculated the 
sensitivity, specificity, PPV, and NPV for both the high EOV signal and the high RHR signal for predicting the primary outcome. All tests were two-sided, and significant differences were considered when $P$ value was less than 0.05. We used $R$ version 3.6.1 ( $R$ Foundation for Statistical Computing, Vienna, Austria) for the analyses.

\section{Data availability}

Anonymized dataset of this study will be available from the corresponding author (anomura@med.kanazawa-u.ac.jp) upon publication. The investigator may only use the data for the purpose outlined in the request. Data redistribution is prohibited.

\section{Code availability}

Any custom code or mathematical algorithm were not used in this study.

\section{Acknowledgements}

We greatly thank all the participants and medical staff, the COVID-19 Special Assistant Team (COVSAT) in Kanazawa University Hospital, Shinichiro Takashima, Hirofumi Okada, Satoshi Niwa, Hayato Tada, Toyonobu Tsuda, many other people in Kanazawa University Hospital and JCHO Kanazawa Hospital for their outstanding work against COVID-19 while simultaneously cooperating with this study. Also, we express our gratitude to Conor Heneghan and Tomoyasu Chigahara for their kind support regarding the EOV analysis. Moreover, we appreciate Jia Yu and Yasuhito Imai for the REDCap maintenance, eConsent production, data cleaning, and data management.

\section{Author contributions}


KY and AN wrote the draft manuscript and have access to all the study data. AN takes responsibility of data integrity and analyzed data. $K Y, A N, M K, M S, K S, S U, K F, M T$, and $M O$ conceptualized and designed this study. KY, AN, and KW collected clinical data from the participants. All the authors approved the final version of the manuscript.

\section{Funding}

The investigational device used in this study, Fitbit Charge 3, was provided by Fitbit Japan.

\section{Competing interests}

The authors declare the following competing interests: AN received consulting fees from the CureApp, Inc. AN was a co-founder of the CureApp Institute. The other authors declare no competing interests. 


\section{References}

1 AFP website, <https://interactive.afp.com/graphics/COVID-19-Rise-in-the-number-ofcases-and-deaths 600/> (Last accessed: Jan 4, 2021)

2 He, X. et al. Temporal dynamics in viral shedding and transmissibility of COVID-19. Nat Med 26, 672-675, doi:10.1038/s41591-020-0869-5 (2020).

3 Arons, M. M. et al. Presymptomatic SARS-CoV-2 Infections and Transmission in a Skilled Nursing Facility. N Engl J Med 382, 2081-2090, doi:10.1056/NEJMoa2008457 (2020).

4 Hu, B., Guo, H., Zhou, P. \& Shi, Z. L. Characteristics of SARS-CoV-2 and COVID-19. Nat Rev Microbiol, doi:10.1038/s41579-020-00459-7 (2020).

5 Richardson, S. et al. Presenting Characteristics, Comorbidities, and Outcomes Among 5700 Patients Hospitalized With COVID-19 in the New York City Area. JAMA, doi:10.1001/jama.2020.6775 (2020).

6 Beigel, J., et al. Remdesivir for the Treatment of Covid-19 - Final Report. N Engl J Med 383, 1813-1826, doi:10.1056/NEJMoa2007764 (2020).

7 Recovery Collaborative Group, et al. Dexamethasone in Hospitalized Patients with Covid-19 - Preliminary Report. N Engl J Med, doi:10.1056/NEJMoa2021436 (2020).

8 Hayasaki, E. Covid-19: how Japan squandered its early jump on the pandemic. BMJ 369, m1625, doi:10.1136/bmj.m1625 (2020).

9 JapanToday. Man in 50s with mild COVID-19 found dead in quarantine hotel room in Kanagawa, <https://japantoday.com/category/national/man-in-50s-with-mild-covid19-found-dead-in-quarantine-hotel-room-in-kanagawa> (2020). (Last accessed: Dec $28,2020)$

10 D'Angelo, B. Coronavirus: 3 men found dead at NYC hotel used to house COVID-19 
patients, <https://www.kiro7.com/news/trending/coronavirus-3-men-found-deadnyc-hotel-used-house-covid-19-patients/APCJSP4FQNAK3HUGIWFPQ6DAVE/> (2020). (Last accessed: Dec 28, 2020)

11 Shah, S., Velardo, C., Farmer, A. \& Tarassenko, L. Exacerbations in Chronic Obstructive Pulmonary Disease: Identification and Prediction Using a Digital Health System. J Med Internet Res 19, e69, doi:10.2196/jmir.7207 (2017).

12 Tobin, M. J., Laghi, F. \& Jubran, A. Why COVID-19 Silent Hypoxemia Is Baffling to Physicians. Am J Respir Crit Care Med 202, 356-360, doi:10.1164/rccm.202006-2157CP (2020).

13 Miller, D., et al. Analyzing changes in respiratory rate to predict the risk of COVID-19 infection. PLoS One 15, e0243693, doi:10.1371/journal.pone.0243693 (2020).

14 Radin, J. M., Wineinger, N. E., Topol, E. J. \& Steinhubl, S. R. Harnessing wearable device data to improve state-level real-time surveillance of influenza-like illness in the USA: a population-based study. Lancet Digital Health 2, e85-93 (2020).

15 Mishra, T., et al. Pre-symptomatic detection of COVID-19 from smartwatch data. Nat Biomed Eng 4, 1208-1220, doi:10.1038/s41551-020-00640-6 (2020).

16 Quer, G., et al. Wearable sensor data and self-reported symptoms for COVID-19 detection. Nat Med, doi:10.1038/s41591-020-1123-x (2020).

17 Natarajan, A., Su, H. W. \& Heneghan, C. Assessment of physiological signs associated with COVID-19 measured using wearable devices. NPJ Digit Med 3, 156, doi:10.1038/s41746-020-00363-7 (2020).

18 Levitan, R. The Infection That's Silently Killing Coronavirus Patients, $<$ https://www.nytimes.com/2020/04/20/opinion/sunday/coronavirus-testing-

pneumonia.html> (2020). (Last accessed: Dec 28, 2020) 
19 Wilkerson, R. G., Adler, J. D., Shah, N. G. \& Brown, R. Silent hypoxia: A harbinger of clinical deterioration in patients with COVID-19. Am J Emerg Med 38, 2243 e2245-2243 e2246, doi:10.1016/j.ajem.2020.05.044 (2020).

20 Couzin-Frankel, J. The mystery of the pandemic's 'happy hypoxia'. Science 368, 455456, doi:10.1126/science.368.6490.455 (2020).

21 Porzionato, A. et al. The potential role of the carotid body in COVID-19. Am J Physiol Lung Cell Mol Physiol 319, L620-L626, doi:10.1152/ajplung.00309.2020 (2020).

22 Semelka, M., Wilson, J. \& Floyd, R. Diagnosis and Treatment of Obstructive Sleep Apnea in Adults. Am Fam Physician 94, 355-360 (2016).

23 Labarca, G., Gower, J., Lamperti, L., Dreyse, J. \& Jorquera, J. Chronic intermittent hypoxia in obstructive sleep apnea: a narrative review from pathophysiological pathways to a precision clinical approach. Sleep Breath 24, 751-760, doi:10.1007/s11325-019-01967-4 (2020). 


\section{Figure legends}

\section{Figure 1. Study flowchart.}

SARS-CoV-2; severe acute respiratory syndrome coronavirus 2; PCR, polymerase chain reaction

Figure 2. Summary of high EOV signals and events among SARS-CoV-2 PCR positive patients. The vertical columns represent ID of each patient, and the horizontal axis represent the number of days after wearing Fitbit. Blue squares represent a single day high EOV signal. Gray columns denote a day of no EOV data obtained. Red columns indicate the days that primary outcomes (symptom exacerbation events) occurred.

EOV, estimated oxygen variations; SARS-CoV-2; severe acute respiratory syndrome coronavirus 2; PCR, polymerase chain reaction

Figure 3. The clinical courses of ID-008, ID-016, and ID-023.

The vertical columns represent EOV, resting heart rate (RHR) (compared with minimum RHR), body temperature, and cough or fatigue severity levels. The horizontal axis represents the number of days after positive on the SARS-CoV-2 PCR test (ID-008) or the occurrences of COVID-19 related symptoms (ID-016 and ID-023).

EOV, estimated oxygen variations; SARS-CoV-2; severe acute respiratory syndrome coronavirus 2; COVID-19, coronavirus disease 2019

Figure 4. Representative graphs of low (negative) and high (positive) EOV signals EOV, estimated oxygen variations 
Figures

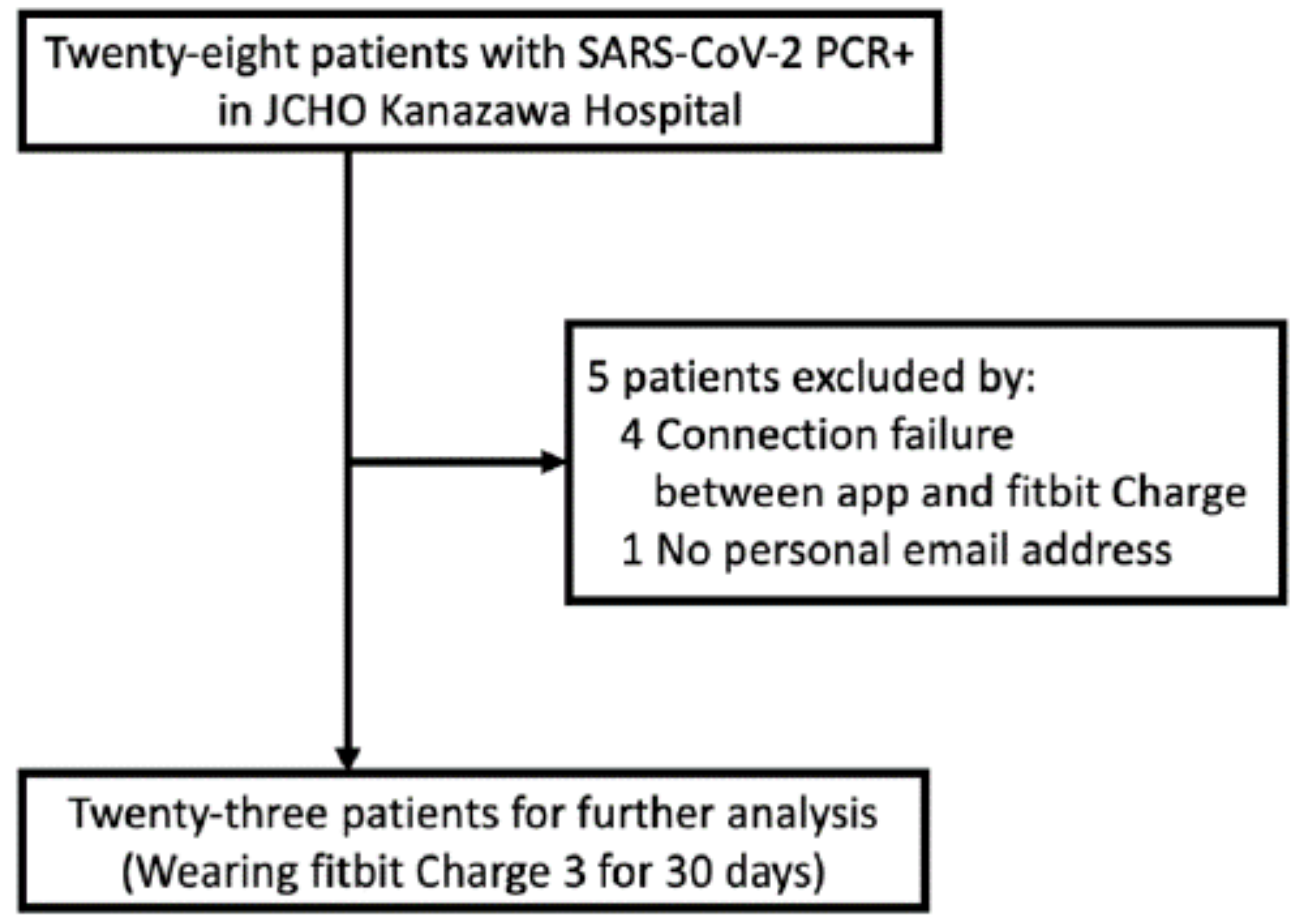

Figure 1

Study flowchart. SARS-CoV-2; severe acute respiratory syndrome coronavirus 2; PCR, polymerase chain reaction 


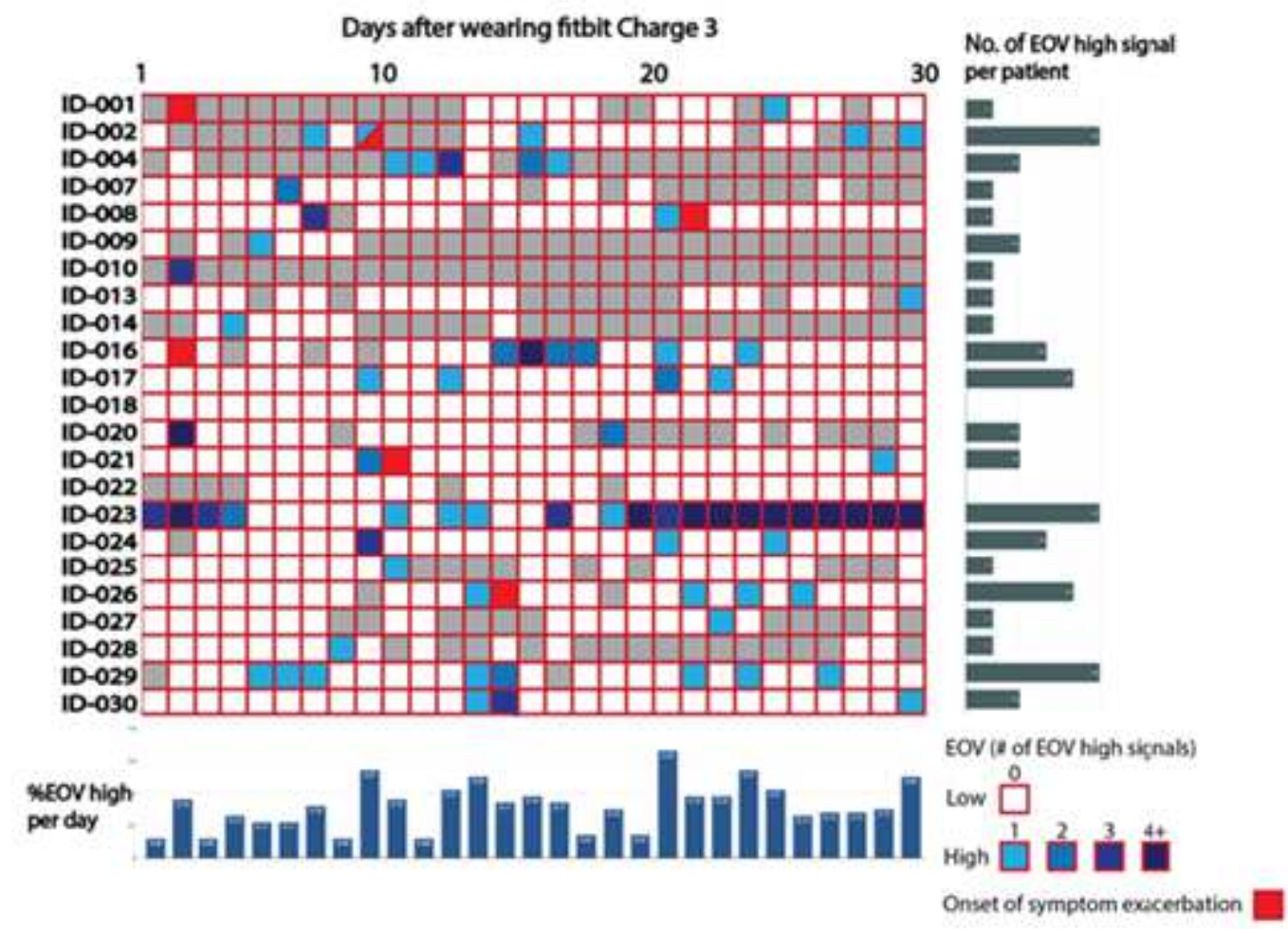

Figure 2

Summary of high EOV signals and events among SARS-CoV-2 PCR positive patients. The vertical columns represent ID of each patient, and the horizontal axis represent the number of days after wearing Fitbit. Blue squares represent a single day high EOV signal. Gray columns denote a day of no EOV data obtained. Red columns indicate the days that primary outcomes (symptom exacerbation events) occurred. EOV, estimated oxygen variations; SARS-CoV-2; severe acute respiratory syndrome coronavirus 2; PCR, polymerase chain reaction 


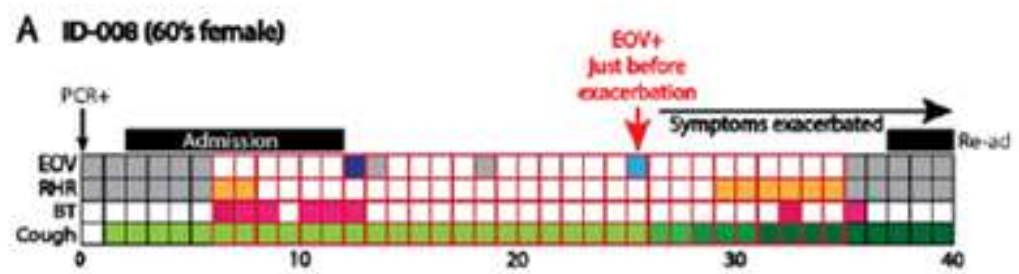

B $10-016$ (70) male)
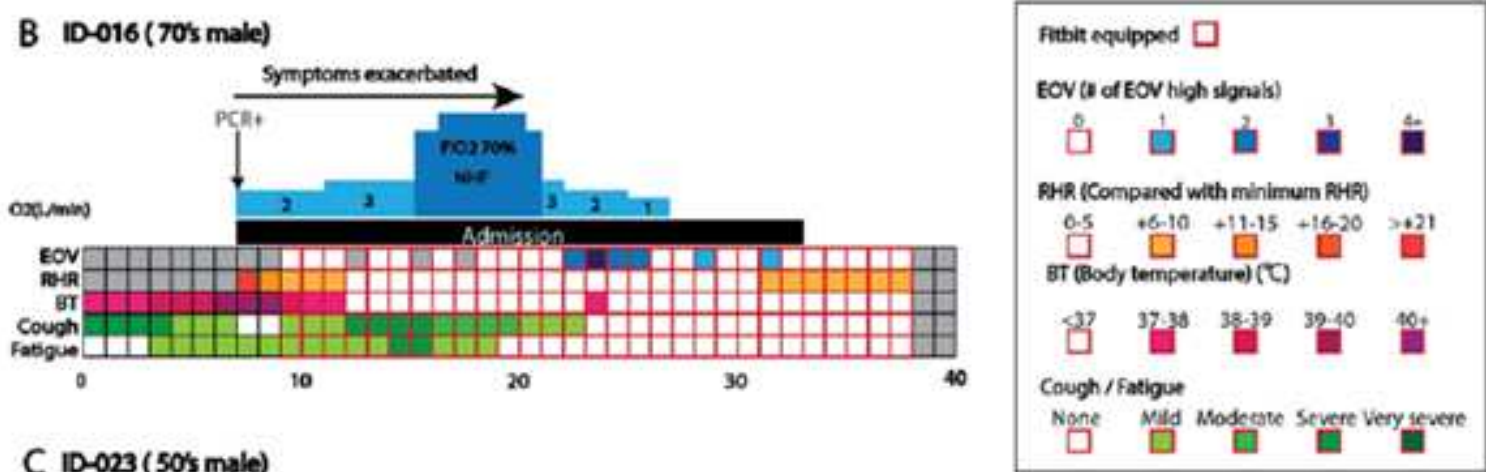

\section{C $10-023$ (50's male)}

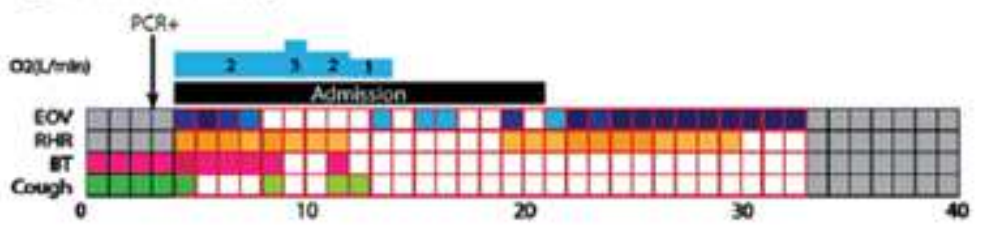

Days ather the onset of inital COVD-19 symptom or SARS-COV-2PCR+

\section{Figure 3}

The clinical courses of ID-008, ID-016, and ID-023. The vertical columns represent EOV, resting heart rate (RHR) (compared with minimum RHR), body temperature, and cough or fatigue severity levels. The horizontal axis represents the number of days after positive on the SARS-CoV-2 PCR test (ID-008) or the occurrences of COVID-19 related symptoms (ID-016 and ID-023). EOV, estimated oxygen variations; SARSCoV-2; severe acute respiratory syndrome coronavirus 2; COVID-19, coronavirus disease 2019

Low

EOV signals

\section{High \\ EOV signals (+)}

No. of high signals
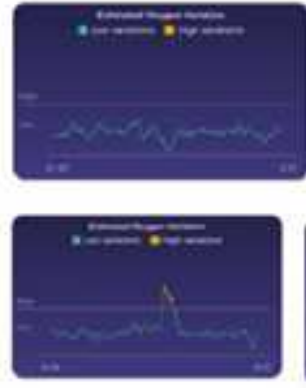

$1+$

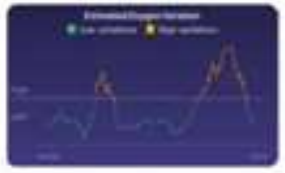

2+

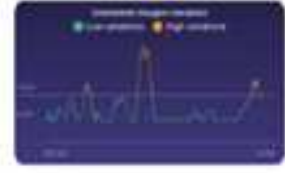

$3+$

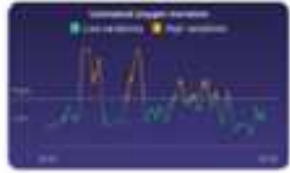

4 or more

Figure 4 
Representative graphs of low (negative) and high (positive) EOV signals EOV, estimated oxygen variations

\section{Supplementary Files}

This is a list of supplementary files associated with this preprint. Click to download.

- Supplementaryinformation.pdf 